\title{
Study of the key problem of geo-information integration and
} sharing platform

\author{
Qingfeng ZHAO ${ }^{1, a}$, Linshan $\mathrm{Ma}^{2, \mathrm{~b}}$ \\ ${ }^{1}$ College of information Science and Engineering, Shandong University of Science and \\ Technology, Qingdao, 266590, China \\ ${ }^{2}$ Hefei University Library, Hefei College, Hefei, 230601, China \\ aemail: zhaoqflldf@163.com, bemail: shawnls@139.com
}

\begin{abstract}
Keywords: Geo-information; Information Integration; Information Sharing; Web Services
\end{abstract}
\begin{abstract}
The article discusses based on metadata and web services geological information integration and sharing service platform architecture. Studies the key problems encountered in the process of platform architecture and the main solution. Article mainly study in the field of information resources as the research object, discusses the meaning and main characteristics of geological information, summarizes the geological information using the major contradictions and problems, and puts forward a geological model of information integration and sharing, was discussed based on metadata and web services geo-information integration and sharing service platform architecture. Studied the main method and the key problems in the process of platform architecture.
\end{abstract}

\section{Introduction}

Current geological information environment, to a greater extent play to the role of the geo-information resources, and better meet the social needs, research and public groups of geo-information demand[1]. from the whole process of geo-information sources to the geo-information using combined with the new theories and methods, to strengthen geo-information integration and sharing of the overall train of thought, constructed based on metadata and geo-information integration and sharing platform of web services [2] [3].

Platform with "summary points - points node - the data resources" three-level structure, adopting "metadata scattered centralized management, data storage" of technology strategy and SOA architecture oriented distributed data sharing platform, the platform can provide users with "one-stop" data sharing service.

Platform during the build process involves several main key problems, the following is the main key problems and solutions of a detailed in this paper [4].

\section{Single sign-on (sso) problem by the user}

Single sign-on (SSO) in use on, refers to the application system for different quickly unified authentication function, achieve global, security software environment. In implementing SSO system, the user for a login operation, can obtain the required access authorization of application systems and resources, don't have to repeatedly enter a user name and password to determine the user's identity, namely "a login, multiple authentication".

In geo-information integration and sharing platform, this paper puts forward a simple solution for single sign-on (sso) and limits of authority, it USES the centralized architecture based on paper, in order to cross domain Cookie sharing as the core to complete the user login, authentication and access control. The basic idea is: in summary points set user registration certification center (Certificate \& Authentication, CA), through the CA unified user register, login, rights management and certification; In each node deployment CA client, by specifying the address of the CA, to join the certification system, configure the access control of resources at the same time. By deploying, 
CA and a summary of points deployed in each node of the CA client, realizing the user's single sign-on (sso) and the effective control of access to resources and certification. Because of the distributed data sharing service system has a lot of nodes, and with the passage of time the data center will be increasing, when the client access to a data center, don't know whether other data nodes in the user login verification.

2.1 Registered users by CA, the CA administrator to classification of user, data center administrators in each node for data classification. Whether the user can access the data resources need to meet the user data access matrix.

2.2 The client access to a data center web site protected resources, single sign-on (sso) module to take over the request first, whether the query client holds the data center of cookies, and attempts to obtain the access of the paper, TokenID. If the client can provide that users ever to legal status to access this site, and has not been cancelled in local, turned to step 5; Otherwise, the following steps.

2.3 The client browser is redirected to the login authentication center, CA is trying to get stored in the client's Cookie, if not, explain global login, the user has not yet turned to step 4; Otherwise, obtained from the Cookie TokenID information user permissions matrix. Then CA will visit the client browser redirects back to the original data center, data center for TokenID and the return address, the TokenID and user permission information written to the client cookies and jump to the return address, and then back to step 2 .

2.4 Redirect the client browser to the login page on the CA, certification center for login user name and password information to check with the user information table, for user identification. Without this user or password mistake, direct return to the login failure; If verification is successful, the only TokenID randomly generated to identify the user login, and generate an entry into the certificate repository in the table, and the user data access matrix information of TokenID along with read into client cookies. The user logs in, turned to step 2 for access to resources.

2.5 Client with TokenID state of user query service to access authentication center, access credentials certification center library table to determine whether the user is still logged in. If the logged in user name and the user's access level to return to the data center, and according to the user's data and user access control to determine whether the user can access data resources. Otherwise, turn to step 4, prompt the user for a user name and password.

\section{3 the problem Of the multi-source information transparent access of foreign}

Geo-information involves different disciplines, different type and format of the data resource, both statistical data, spatial data, and multimedia data, both in the storage form of structured data table, web resources, and file storage of data. Detail see Figure (a).In order to allow the user to the earth system science data sharing in distributed online transparent access to these heterogeneous data resources, put forward and designed a "data services" [5] [6].

"Data services" is a kind of abstract encapsulation of heterogeneous data resources, according to the type of data resources and deposit in the form of different, can be divided into: file service instance, database services, geo-information geographic information service instance, HTTP service instance, FTP service and grid service instances. After the data producer concurrent metadata, need concurrent corresponding instances of data services, such as spatial data, and can be released into the file service instance or geo-information service instance, and then to bind data service and metadata (a metadata can bind more data service instance) [7]. When the data users search for metadata, click "data access", according to the metadata site identification, calls to display the corresponding site data binding with the metadata service instance, user data according to different needs to access the service instance. Such as click file service instance directly download data or click browse online database service instance data in a database table or click on the geo-information service. 


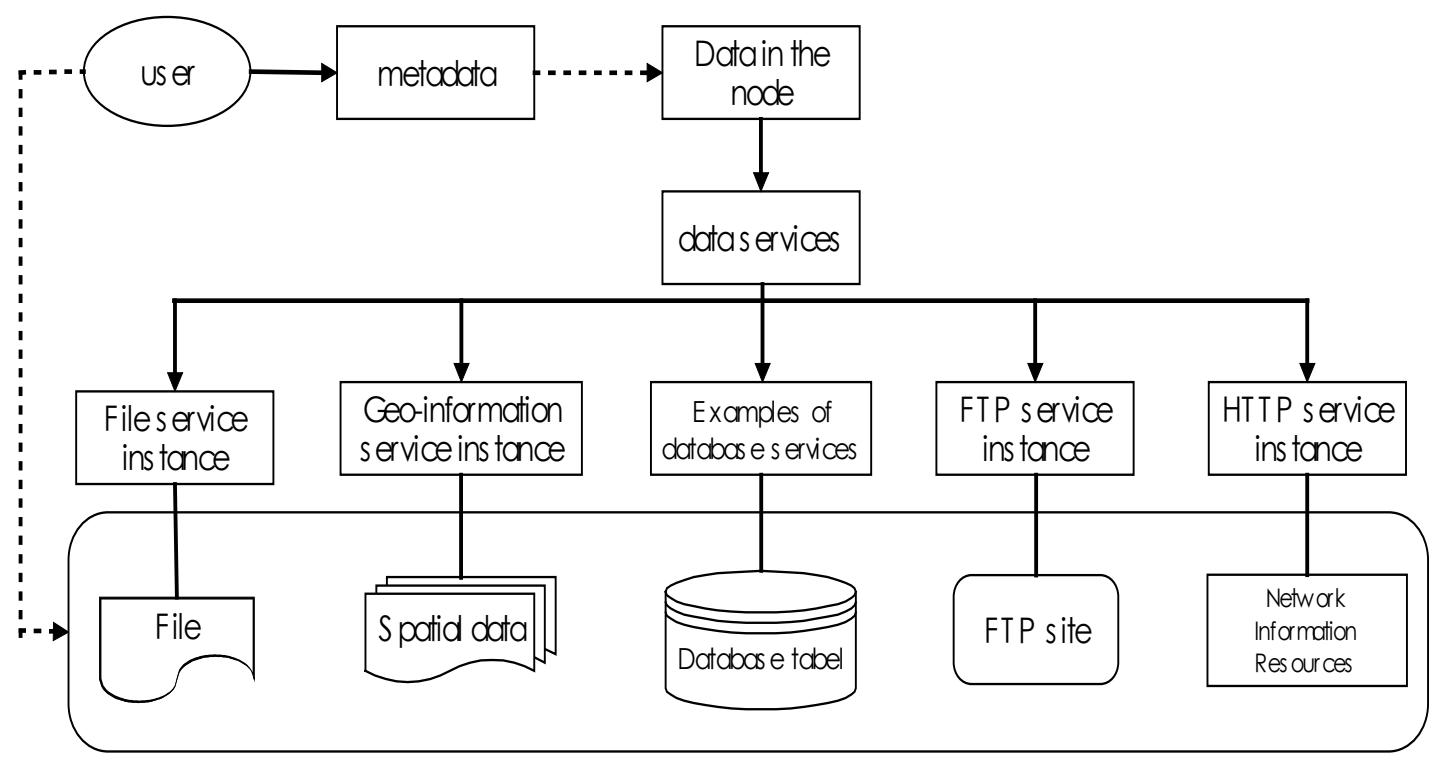

(a) Multi-source remote access to information flow

\section{The problem of Platform for condition monitoring}

Condition monitoring refers to the service platform in the network node using some independent process, continuously, automatically detect the current service platform on a regular basis each component of the running state, the state by SMS, E-mail in the form of a release to operations staff. When there is more severe situation platform, checking program will call the police. Inspection procedure enhance the automation of the operational platform, is the guarantee platform $7 \times 24$ hours normal operation of the necessary means. The checking including server status checking and core service platform service inspection and inspection service interface.

4.1 The server status checking

Server status checking refers to the hardware, operating systems and network infrastructure, such as inspection, its function mainly includes the performance of a data sampling system, the database server, and monitoring system of three parts[8]. Among them, the data sampling system is responsible for gathering the data sent to the database server, database server is responsible for the storage, provide performance data, the data of database performance monitoring system is responsible for the analysis summary, and will feedback the results to the user, alarm processing when necessary[9][10].

From the server in the system for each index of resources of the system information, including CPU usage, memory usage, disk usage of each partition, ICMP, etc., and the data transfer to the database server.

Server performance data browsing program is mainly responsible for the performance index (such as CPU usage, memory usage, ICMP, etc.) to extract and display the information of data. At the same time, the performance index of abnormal alarm processing.

4.2 The Core services inspection

The Core services inspection is important to constitute the basic platform of internal inspection services, these services are the core parts of the platform, the service problems, may lead to foreign most can't even normal access to all the service interface.

In the actual operation process, to set the checking delay interval, in seconds to Oracle, core services such as WMS, WFS, check and monitor the running state.

4.3 The service interface checking

Service interface checking refers to the service of checking, this kind of service is called external users directly. To test the service cluster of various services, including parameter configuration, start, stop, review inspection. Mainly by setting the host IP and port number and inspection time delay, check regularly, and safe information or fault information sent to the designated mobile phone 
number.

\section{The summary}

The framework to solve the traditional information integration cannot solve the problem of semantic heterogeneity, achieve the transparent access of information and the information integration of original system, satisfy the information integration of dynamic, platform independence and scalability requirements. Above work is only in a preparatory stage, the further geo-information integration and sharing platform on the basis of deep geo-information to meet the demand and there are many problems need to solve, I believe that geo-information mining and decision support is in a period of time the mainland a hot issue in the field of information management. So I will try to solve the following problems in the future research and work.

\section{Acknowledgement}

In this paper, the research was sponsored by the Shandong university of science and technology talent introduction research start funded projects (Project No. 2014RCJJ050).

\section{References}

[1]Kyoungjin Park, Yilmaz, Alper.A Design of Web Service for Digital Photogrammetry Workstation using Service Oriented Architecture[J]. AIP Conference Proceedings.2008(1) 204-207.

[2] Goodall, Jonathan L, Horsburgh, Jeffery S, Whiteaker, Timothy L, Maidment, David R, Zaslavsky, Ilya. A first approach to web services for the National Water Information System[J]. Environmental Modelling \& Software. 2008, 23(4) 404-411.

[3] Barlow, Alexis, Li Feng.Online value network linkages: integration, information sharing and flexibility[J]. Electronic Commerce Research \& Applications. 2005, 4(2) 100-112.

[4] Tschangho John Kim. Metadata for geo-spatial data sharing: A comparative analysis[J]. The Annals of Regional Science.1999,(33) 171-181.

[5] Southwick,Silvia B,Lampert,Cory. Metadata Dictionary Database: A Proposed Tool for Academic Library Metadata Management[J]. Journal of Electronic Resources Librarianship.2011,23(4) 339-360.

[6] Oskou'ee, A. Kalantari.Geo-metadata creation using GML Technology [J]. International Journal of Information Science \& Technology. 2007, 5(2) 5.

[7] Kim, Tschangho John. Metadata for geo-spatial data sharing: A comparative analysis[J]. Annals of Regional Science. 1999, 33(2) 171-181.

[8] Yue Peng,Gong Jianya,Di Liping.Augmenting geospatial data provenance through metadata tracking in geospatial service chaining[J]. Computers \& Geosciences. 2010, 36 (3) 270-281.

[9] Li Xiaoyan, Di Liping, Han Weiguo, Zhao Peisheng, Dadi, Upendra. Sharing geoscience algorithms in a Web service-oriented environment (GRASS GIS example) [J]. Computers \& Geosciences. 2010,36(8) 1060-1068.

[10] Nogueras-Iso J, Zarazaga-Soria, F.J, Lacasta, J. Béjar, R. Muro-Medrano, P.R..Metadata standard interoperability: application in the geographic information domain[J]. Computers, Environment \& Urban Systems. 2004, 28(6) 611-634. 